\title{
Kommentar II zum Fall: ,Therapiezieländerung bei einem Kind mit schweren angeborenen Fehlbildungen“"
}

\author{
Julia Inthorn
}

Angenommen: 2. November 2021 / Online publiziert: 16. November 2021

(C) Der/die Autor(en) 2021

Der vorgestellte Fall kann hinsichtlich der ethischen Fragen ${ }^{1}$ aus zwei Blickrichtungen betrachtet werden: Zum einen ist hier der - auch in der abschließenden Frage formulierte - Status der Abwägung von Nutzen und Schaden gegenüber dem Lebensrecht des Kindes zu nennen, zum anderen die Frage des Vorgehens in der gemeinsamen Entscheidungsfindung.

Der Verweis auf das Lebensrecht des Kindes hat als Argument im Prozess einer gemeinsamen Entscheidungsfindung in der Regel eine andere Funktion als abwägende Argumente bei der Bewertung von Nutzen und Schaden. Durch den Verweis auf das Lebensrecht wird die grundsätzliche Frage aufgeworfen, wie weit Eltern (ebenso wie andere Personen) Entscheidungen treffen dürfen, die das Versterben des Kindes zur Folge haben (können), und damit, welche Spielräume in Abwägungen insbesondere bei Neugeborenen wie im vorliegenden Fall ethisch zulässig sind. Aus ethischer Perspektive sind die Pflichten insbesondere von Eltern und Ärzt*innen in einer Entscheidungssituation wie der vorgestellten eng damit verwoben, was die Inhalte der Abwägung sind.

Ist die Abwägung von Nutzen und Schaden aus medizinischer Sicht eindeutig, ist dieses Ergebnis ethisch maßgeblich und für die Entscheidung leitend. Dies kann der Fall sein, wenn eine Maßnahme mit nahezu $100 \%$ iger Wahrscheinlichkeit dazu führt, dass das Kind wieder vollständig gesund wird, oder auch wenn alle kurativen Möglichkeiten ausgeschöpft sind. Eine solche Eindeutigkeit scheint hier nicht gegeben zu sein, es bestehen weitere kurative Optionen, allerdings wird für diese

\footnotetext{
${ }^{1}$ Der Kommentar beschränkt sich auf die ethische Perspektive, eine Einschätzung aus rechtlicher Sicht kann hier nicht geleistet werden.
}

Dr. Julia Inthorn $(\bowtie)$

Zentrum für Gesundheitsethik (ZfG), Knochenhauerstr. 33, 30159 Hannover, Deutschland

E-Mail: julia.inthorn@evlka.de 
eine minimale Wahrscheinlichkeit des Überlebens, ein hohes Risiko des Eingriffs und weitergehende Probleme angegeben.

Ist die Abwägung von Nutzen und Schaden medizinisch weniger eindeutig, etwa weil mit (schweren) Komplikationen bei einem Eingriff gerechnet werden muss, wächst damit die Aufgabe, diese Abwägung im Prozess der gemeinsamen Entscheidungsfindung im Sinne des Kindes zu treffen. Hierzu sollten die verschiedene Therapieziele und Handlungsoptionen offen benannt werden. Allein die Tatsache, dass das Versterben des Kindes bei einer Handlungsoption möglich oder sogar wahrscheinlich ist, sollte nicht dazu führen, dass diese Handlungsoption aus der Bewertung ausgeschlossen wird. Vielmehr ist für jede Option das Nutzen-Schadens-Verhältnis abzuwägen. Diese Abwägung beinhaltet dabei immer Bewertungen, die weit über eine medizinische Risikoanalyse hinausgehen und in die Aspekte wie die Lebensqualität des Kindes, die familiäre Situation in der Versorgung durch die Eltern oder die Entwicklungschancen des Kindes einfließen können. Im Rahmen der gemeinsamen Entscheidungsfindung (Shared decision making) sollten die Eltern von ärztlicher Seite dabei unterstützt werden, ihre Vorstellungen und Gewichtungen der Dimensionen vom Wohl des Kindes auf die konkrete Entscheidungssituation und die Bewertung von Nutzen und Schaden medizinischer Maßnahmen zu übertragen. ${ }^{2}$ Die Eltern werden dabei versuchen, ihrer Vorstellung von Familie und elterlicher Fürsorge bestmöglich gerecht zu werden. Dabei spielt in der Regel auch eine Rolle, welche Vorstellung sie davon entwickeln können, wie sie ihr Kind in der jeweiligen Situation begleiten und unterstützen können. Diese Fürsorge ist auch für die weitere Behandlung ein wesentlicher Baustein.

In der geschilderten Situation bestehen Behandlungsoptionen, die jeweils auf die einzelnen Symptome ausgerichtet sind. Eine Schwierigkeit der Abwägung besteht in der schwer einschätzbaren Kombination der Symptome und deren wechselseitiger Einfluss, insbesondere hinsichtlich der zu erwartenden Komplikationen und zusätzlichen Belastungen für das Kind bei einer Operation des Speiseröhrenverschlusses bei gleichzeitig minimaler Erfolgsaussicht. Die Abwägung und gemeinsame Entscheidung von Ärzt:innen und Eltern scheint mit viel Bedacht und großer Sorgfalt geführt worden zu sein. Welche weiteren Behandlungsoptionen und möglichen Therapieziele dabei diskutiert wurden ist nicht bekannt. Diese Sorgfalt vorausgesetzt, ist die Entscheidung ethisch nachvollziehbar - hätten andere Eltern in einer solchen Situation anders entschieden, wäre aber auch dieser Ausgang ethisch begründbar.

Das wirft die Frage auf, woher das moralische Unbehagen auf ärztlicher Seite rührt, den gemeinsam entschiedenen Weg mitzutragen. Der Entscheidung im geschilderten Fall bezüglich des Therapieziels ging bereits eine Auseinandersetzung mit der Frage der Abtreibung voraus. Die Eltern hatten sich im Verlauf der Schwangerschaft mit Fragen des Lebens mit einem behinderten Kind auseinandergesetzt. Über die Überlegungen auf Seiten der Eltern bei ihrer Entscheidung während der Schwangerschaft ist wenig bekannt. Berichtet wird, dass die Eltern nach dem Ge-

\footnotetext{
2 Weitere auf diese Situation übertragbare Hinweise zum Prozess der Entscheidungsfindung finden sich in der AWMF Leitlinie „Frühgeborene an der Grenze der Lebensfähigkeit“, abrufbar unter: https://www. awmf.org/uploads/tx_szleitlinien/024-0191_S2k_Fr\%C3\%BChgeburt_Grenze_Lebensf\%C3\%A4higkeit_ 2021-01.pdf (zugegriffen: 29. Okt. 2021).
} 
spräch in der pränatalmedizinischen Praxis keine weiteren Informationen einholten, die Option eines Schwangerschaftsabbruchs nicht weiter verfolgten und im Verlauf der weiteren Schwangerschaft eine neue Vorstellung von sich als Familie („Leben mit einem behinderten Kind“) entwickelt haben. Der Abwägungsprozess mit den Eltern zur Festlegung des Therapieziels beim geborenen Kind nach Abschluss aller Diagnostik wird als gemeinsame Entscheidungsfindung nach wiederholten Gesprächen charakterisiert, hier entscheiden die Eltern sich für das palliative Therapieziel. Dass von ärztlicher Seite diese gemeinsame Entscheidung dennoch nachträglich in Frage gestellt wurde, kann sehr unterschiedliche Hintergrunde haben, die nicht weiter bekannt sind, und zeigt, dass im Gespräch zwischen Eltern und Ärzt:innen nicht alle ethisch relevanten Aspekte benannt und Bedenken ausgeräumt werden konnten. Eine klinisch-ethische Fallbesprechung hätte den Ärzt:innen hier die Möglichkeit geben können, eigene moralische Fragen anders in einer Entscheidungsfindung vortragen zu können als es im Prozess der gemeinsamen Entscheidungsfindung ohne Moderation möglich ist.

Funding Open Access funding enabled and organized by Projekt DEAL.

Open Access Dieser Artikel wird unter der Creative Commons Namensnennung 4.0 International Lizenz veröffentlicht, welche die Nutzung, Vervielfältigung, Bearbeitung, Verbreitung und Wiedergabe in jeglichem Medium und Format erlaubt, sofern Sie den/die ursprünglichen Autor(en) und die Quelle ordnungsgemäß nennen, einen Link zur Creative Commons Lizenz beifügen und angeben, ob Änderungen vorgenommen wurden.

Die in diesem Artikel enthaltenen Bilder und sonstiges Drittmaterial unterliegen ebenfalls der genannten Creative Commons Lizenz, sofern sich aus der Abbildungslegende nichts anderes ergibt. Sofern das betreffende Material nicht unter der genannten Creative Commons Lizenz steht und die betreffende Handlung nicht nach gesetzlichen Vorschriften erlaubt ist, ist für die oben aufgeführten Weiterverwendungen des Materials die Einwilligung des jeweiligen Rechteinhabers einzuholen.

Weitere Details zur Lizenz entnehmen Sie bitte der Lizenzinformation auf http://creativecommons.org/ licenses/by/4.0/deed.de.

Interessenkonflikt J. Inthorn gibt an, dass kein Interessenkonflikt besteht. 